adolescence while that in female twins decreases. Pre- and perinatal insults were not the explanation for an increased incidence of ADHD among twins.

\title{
COGNITIVE FUNCTION AND VALPROATE MONOTHERAPY
}

A test battery to assess neuropsychological and behavioral changes associated with anticonvulsant, particularly valproate, therapy in children is proposed from the Departments of Pediatrics (Neurology), and Clinical Health and Psychology, University of Florida, Gainesville, FL. This includes 1) intellectual functioning (WISC-III, WPPSI-R), 2) verbal memory, sentence recall, story recall, and verbal learning (Wide Range Assessment of Memory and Learning-WRAML, 3) nonverbal memory, picture memory and visual learning-WRAML, 4) attention, digit span, continuous performance task-Paced Auditory Serial Addition Task-PASAT, 5) motor speed-finger tapping test, verbal fluency-Controlled Oral Word Association, and 6) problem behaviorsChild Behavior Check List. These tests were found to be sensitive to AEDinduced cognitive changes, and some tests are repeatable to allow for frequent monitoring. (Legarda SB et al. Altered cognitive functioning in children with idiopathic epilepsy receiving valproate monotherapy. I Child Neurol July 1996;11:321-330). (Respond: Dr Stella B Legarda, Division of Neurology, Department of Pediatrics, University of Florida College of Medicine, PO Box 100296, JHM Health Center, Gainesville, FL 32610).

COMMENT. The authors comment that the cognitive effects of valproate reported in normal adult volunteers and adults with epilepsy cannot reliably be applied to children. There is a relative paucity of well-controlled studies assessing memory and attentional differences in pediatric epilepsy patients treated with valproate monotherapy. Reports that cognitively impaired children on valproate therapy improve with L-acetylcarnitine supplements requires further study.

In one study involving children with epilepsy previously untreated, significant positive correlations were found between serum levels of valproate and the sum of 5 memory tests at 1 month and at 6 months after starting valproate monotherapy. Phenytoin had no adverse effects, whereas carbamazepine serum levels showed a negative correlation with memory and reading scores. (Forsythe I et al. Dev Med Child Neurol 1991;33:524). For reviews of Cognitive Effects of Antiepileptic Drugs, see Progress in Pediatric Neurology II, Chicago, PNB Publ, 1994.

\section{TOURETTE'S SYNDROME AND ADHD}

\section{CORPUS CALLOSUM SIZE IN TOURETTE'S SYNDROME}

The size of the corpus callosum (CC) in Tourette's syndrome (TS) and ADHD was determined by analysis of MRI data in 77 children and adolescents, aged 6 to 16 years, including 27 controls, at the Kennedy Krieger Institute, Johns Hopkins University School of Medicine, Baltimore, MD. TS patients had significant increases in 4 of 5 subregions (splenium, isthmus/posterior body, mid-body, and rostral body), the total area, and the perimeter of the CC. ADHD was associated with a significant decrease in the rostral body size. Inspection of subgroup means demonstrated a statistical independence of the effects of ADHD versus effects of TS on CC size. The larger CC in TS was independent of age, handedness, intracranial area, and the association of ADHD. (Baumgardner TL, Singer HS, Denckla MB et al. Corpus callosum morphology in 